\title{
Programa com jogos de raciocínio para adolescentes em situação de dependência de substâncias psicoativas
}

\author{
Cláudio Elias Duarte ${ }^{1}$ \\ (D) https://orcid.org/0000-0002-8310-2058 \\ Sandra Regina Rezende Garcia² \\ (D) https://orcid.org/0000-0002-2109-411X \\ Sarah Virginia dos Santos Braga ${ }^{2}$ \\ (D) https://orcid.org/0000-0001-9161-2149 \\ Felipe Salles Neves Machado ${ }^{3}$ \\ (D) https://orcid.org/0000-0002-3850-359X \\ Jefferson Luiz Pereira ${ }^{4}$ \\ (D) https://orcid.org/0000-0002-7610-467X \\ Sofia Marques Viana Ulisses ${ }^{4}$ \\ (D) https://orcid.org/0000-0002-7128-3672
}

${ }^{1}$ Hospital Santa Mônica, Unidade Itapecerica, Itapecerica da Serra, SP, Brasil.

2 Mind Lab do Brasil, Unidade Vila Mariana, São Paulo, SP, Brasil.

${ }^{3}$ Centro de Atenção Integrada à Saúde Mental Philippe Pinel, Unidades de Pirituba, São Paulo, SP, Brasil.

${ }^{4}$ Pontifícia Universidade Católica de São Paulo, Campus Perdizes, São Paulo, SP, Brasil
Objetivo: verificar impactos da aplicação de um programa com uso de jogos de raciocínio embasado na metodologia Mind $L a b$ nas funções executivas atenção, flexibilidade e planejamento em adolescentes em situação de dependência de substâncias psicoativas. Método: estudo quantitativo quase-experimental com aplicação de instrumentos de avaliação neuropsicológica pré e pós-intervenção. Participaram 35 adolescentes com idades entre 12 e 17 anos internados em um Centro de Atenção Integrada à Saúde Mental na cidade de São Paulo. Resultados: evidenciou-se melhora na atenção após a aplicação do programa com jogos. Quanto às funções planejamento e flexibilidade, foram observadas melhoras, porém estas não foram significativas. Conclusão: foi possível verificar a importância da utilização de jogos de raciocínio como recurso lúdico no tratamento de adolescentes em condição de dependência de substâncias psicoativas, possibilitando a construção de estratégias que auxiliem no processo de recuperação e reabilitação.

Descritores: Adolescentes; Função Executiva; Metacognição; Dependência de Substâncias Psicoativas.

\section{Como citar este artigo}

Duarte CE, Garcia SRR, Braga SVS, Machado FSN, Pereira JL, Ulisses SMV. A thinking games program for adolescents in a situation of psychoactive-substance dependence. SMAD, Rev Eletrônica Saúde Mental Álcool Drog. 2021 abr.-jun.;17(2):54-63. doi: https://dx.doi.org/10.11606/issn.1806-6976.smad.2021.166885 


\title{
A thinking games program for adolescents in a situation of psychoactive-substance dependence
}

\begin{abstract}
Objective: to analyze the impacts of the application of a program using thinking games based on the Mind Lab methodology on the executive functions: attention, flexibility and planning in adolescents in a situation of psychoactive-substance dependence. Method: quasi-experimental quantitative study with the application of neuropsychological assessment tools before and after intervention. Thirty-five adolescents aged 12 to 17 years old, admitted to an Integrated Mental Health Care Center in the city of São Paulo, participated in this study. Results: there was evidence of attention improvement after application of the games program. Regarding the planning and flexibility functions, improvements were observed, but they were not significant. Conclusion: it was possible to observe the importance of using thinking games as a playful resource in the treatment of substance-dependent adolescents, thus enabling the construction of strategies that assist in their recovery and rehabilitation process.
\end{abstract}

Descriptors: Adolescents; Executive Function; Metacognition; Substance Dependence.

\section{Programa con juegos de pensamiento para adolescentes en situación de dependencia a sustancias psicoactivas}

\begin{abstract}
Objetivo: verificar impactos de la aplicación de un programa utilizando juegos de pensamiento basados en la metodología Mind Lab sobre las funciones ejecutivas de atención, flexibilidad y planificación en adolescentes en situación de dependencia a sustancias psicoactivas. Método: estudio cuantitativo cuasiexperimental con aplicación de instrumentos de evaluación neuropsicológica antes y después de la intervención. Treinta y cinco adolescentes de 12 a 17 años, ingresados en un Centro Integrado de Atención de Salud Mental en la ciudad de São Paulo, participaron en este estudio. Resultados: hubo evidencia de mejora en la atención después de la aplicación del programa con juegos. Con respecto a las funciones de planificación y flexibilidad, se observaron mejoras, pero estas no fueron significativas. Conclusión: fue posible verificar la importancia del uso de juegos de pensamiento como recurso lúdico en el tratamiento de adolescentes en condición de dependencia de sustancias psicoactivas, permitiendo la construcción de estrategias que ayuden en el proceso de recuperación y rehabilitación.
\end{abstract}

Descriptores: Adolescentes; Función Ejecutiva; Metacognición; Dependencia a Sustancias. 


\section{Introdução}

A dependência de substâncias psicoativas (SPAs) consiste em alterações comportamentais, fisiológicas e cognitivas, caracterizando um quadro de saúde mental descrito pelo consumo frequente, compulsivo, destinado à evitação de sintomas de abstinência e acompanhado por problemas físicos, psicológicos e sociais ${ }^{(1-2)}$. Trata-se de um problema que demanda atenção especial na adolescência, por ser esta uma trajetória crítica do desenvolvimento. É nesse contexto que se inserem preocupações associadas ao consumo de SPAs nessa fase da vida, haja vista que as consequências decorrentes desse consumo podem ser mais danosas do que aquelas observadas na vida adulta - isto porque, de um lado há especificidades existenciais dessa trajetória da vida, e de outro, questões neuroquímicas em função do amadurecimento cerebral(3-4).

Diferentemente de um adulto, o cérebro do adolescente ainda está em processo de desenvolvimento, com diversas regiões em formação, como é o caso das estruturas responsáveis pelo chamado cérebro social e várias outras que fazem parte do circuito de recompensa ${ }^{(5-6)}$. Apesar de o cérebro em desenvolvimento ser mais resiliente a efeitos neurotóxicos, o consumo de álcool e outras substâncias durante um período crítico do desenvolvimento neurológico pode interromper o curso natural de maturação do cérebro e de processos-chave do seu desenvolvimento.

Prejuízos nas funções executivas foram associados aos riscos e consequências da dependência de SPAs em estudos prévios ${ }^{(7-9)}$, destacando-se prejuízos nos domínios da atenção, memória, comportamentos (e.g. impulsividade) e afetividade. As funções executivas envolvem um amplo espectro de competências comportamentais e habilidades cognitivas que constituem importantes recursos para a otimização de respostas aos desafios ambientais e pessoais, a fim de maximizar oportunidades de recompensa e alcançar metas de longo prazo $^{(9)}$. Intervenções voltadas para o desenvolvimento de tais funções podem ser promissoras no tratamento e reabilitação de pacientes com quadros de dependência em SPAs ${ }^{(10)}$, notadamente para adolescentes, pois se de um lado operam fatores de risco relativos ao seu desenvolvimento neural e cognitivo, de outro há aspectos protetivos que esses mesmos atributos podem promover - capacidade de adaptação e flexibilidade do cérebro (plasticidade neural)(11).

A utilização de jogos de raciocínio como recurso no tratamento de adolescentes dependentes de SPAs ancora-se no pressuposto de que a metodologia Mind $L a b^{(10)}$, criada para o ambiente educacional, quando aplicada no contexto clínico, poderia colaborar para uma melhora das funções executivas. Jogos são recursos privilegiados para explorar diferentes aspectos cognitivos e sociais, pois possibilitam trabalhar com regras e estratégias em diferentes contextos relacionais. Por meio de jogos de raciocínio é possível exercitar atitudes de respeito à vez do outro, análise de dados, bem como funções de atenção, flexibilidade, planejamento e tomada de decisões ${ }^{(12)}$. Na metodologia proposta, os participantes aprendem métodos metacognitivos desenvolvidos pela Mind $L a b$, os quais são aplicados na situação de jogo e transferidos, por meio das ações intencionais do mediador, para o enfrentamento das situações do dia a dia(10).

A presente pesquisa é relevante na produção de conhecimento na área da saúde mental, possibilitando ampliar os recursos interventivos para recuperação e reabilitação do adolescente em situação de dependência de SPAs. Até onde nos foi dado a conhecer, poucos estudos versaram sobre intervenções direcionadas à promoção de melhoras nas funções executivas dessa população. Igualmente, a utilização de mediações intencionais, por meio de jogos de raciocínio como recurso no tratamento de pacientes com quadros de dependência de SPA, é ainda incipiente ${ }^{(10)}$.

Assim sendo, o presente estudo teve por objetivo verificar impactos da aplicação de um programa com uso de jogos de raciocínio embasado na metodologia Mind $L a b$ nas funções executivas atenção, flexibilidade e planejamento em adolescentes em situação de dependência de substâncias psicoativas internados para tratamento medicamentoso em um Centro de Atenção Integrada à Saúde Mental (CAISM) na cidade de São Paulo.

\section{Método}

O método proposto para este estudo ancora-se numa perspectiva quantitativa, tratando-se de um estudo de desenho quase-experimental com pré e pós-teste. Tal metodologia investigativa é indicada para estudos nos quais o método experimental é impossibilitado devido ao difícil acesso ao problema ou população investigada(13). A aplicação dessa estratégia metodológica se adequa ao trabalho proposto uma vez que o estudo com a população investigada foi descrito previamente como desafiador, pois os adolescentes demonstram alto nível de resistência, dificuldades de adesão ao tratamento e altas taxas de abandono ${ }^{(14-17)}$.

A pesquisa consistiu em duas etapas. A primeira delas teve como objetivo caracterizar a população segundo o consumo de SPAs, e a segunda, avaliar os impactos da metodologia Mind Lab, utilizando jogos de raciocínio nas funções de atenção, planejamento e flexibilidade. Ambas as etapas foram realizadas no Núcleo da Infância e Adolescência (NIA) do CAISM Philippe Pinel na cidade de São Paulo. O NIA atende crianças e adolescentes, apenas do sexo masculino, com quadros de dependência em SPAs. Há doze leitos disponíveis nessa clínica e o protocolo de internação é de um período médio de trinta dias, apesar de muitos pacientes receberem altas antes desse intervalo de tempo. 
Da primeira etapa participaram trinta e cinco adolescentes que estavam internados na instituição no período da coleta de dados e que preencheram os seguintes critérios de inclusão: ter entre 12 e 17 anos; não apresentar comorbidade psiquiátrica descompensada (sintomas psicóticos ativos, polarização de humor grave, agitação psicomotora, rebaixamento cognitivo severo). Para a segunda etapa da pesquisa foram considerados apenas os adolescentes que participaram de pelo menos $50 \%$ das sessões de intervenção com os jogos e realizaram todos os testes pré e pós intervenção.
Dos trinta e cinco adolescentes que participaram da primeira etapa do estudo e iniciaram a participação nas sessões com jogos, alguns receberam alta durante a intervenção ou antes da aplicação dos testes em pós intervenção. Outro motivo de perda de participantes foi a invalidação de alguns instrumentos devido à recusa em concluí-los. A Figura 1 ilustra o desenho metodológico e o número de participantes em cada etapa da pesquisa e por teste de avaliação neuropsicológica antes e após a intervenção (T0 e T1, respectivamente).

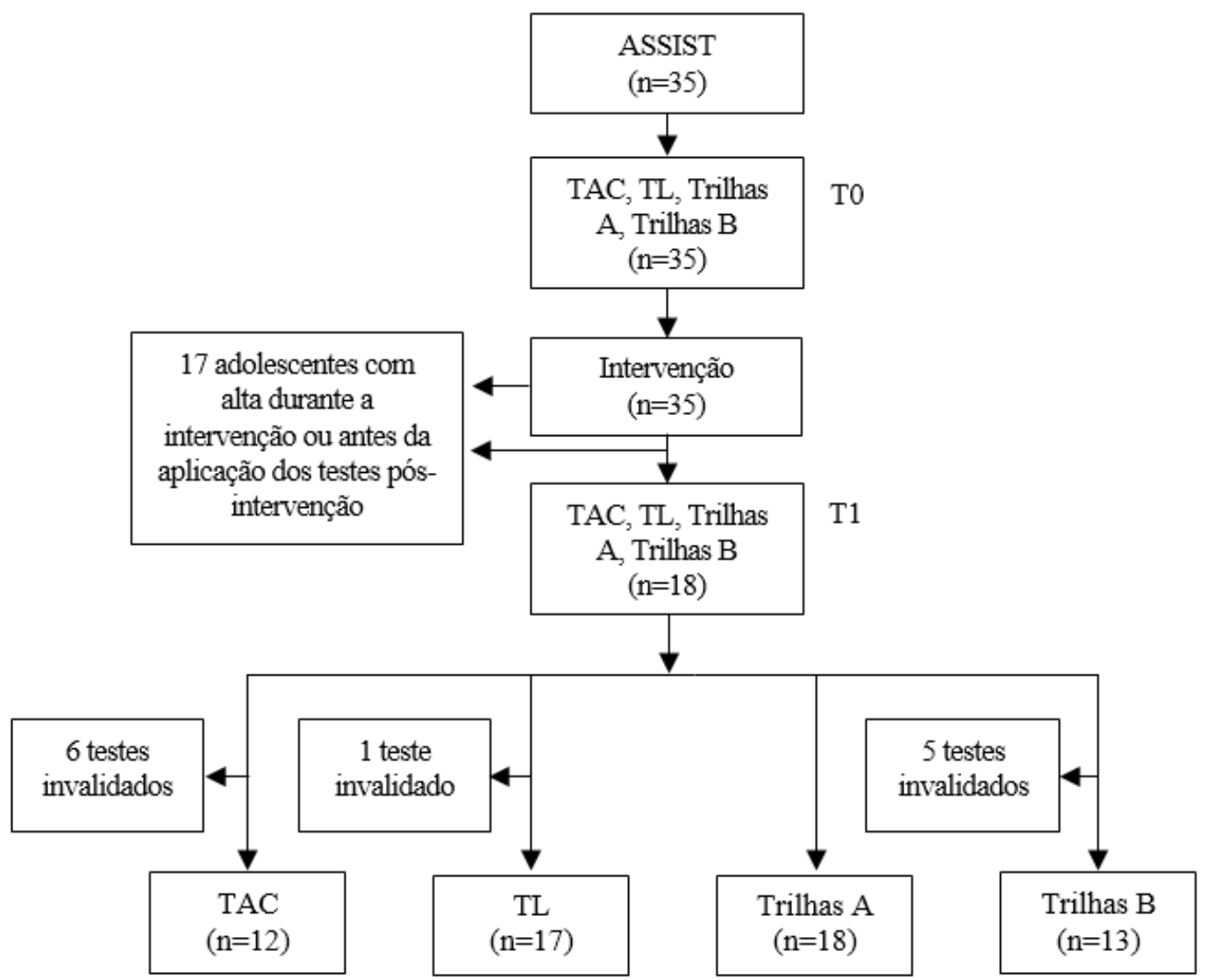

Figura 1 - Desenho metodológico e número de participantes por tipo de teste

A primeira bateria de avaliações (TO) ocorria pelo menos 72 horas após a internação do adolescente no NIA (passado o pico de desintoxicação), e a intervenção tinha início na segunda semana de internação dos participantes. Como a data de entrada dos adolescentes no NIA variou, foram formados seis grupos para a participação nas sessões com jogos de raciocínio utilizando a metodologia Mind Lab, que ocorreram no período de setembro de 2017 a agosto de 2018, conforme descrito na Tabela 1.
Tabela 1 - Descrição dos grupos e período das intervenções em 35 adolescentes internados no CAISM Philippe Pinel, no período de 2017 a 2018. São Paulo, SP, Brasil, 2018

\begin{tabular}{cccc}
\hline Grupo & Período da intervenção & n em T0 & n em T1 \\
\hline 1 & 18 a 29/09/2017 & 10 & 6 \\
2 & 19 a 30/03/2018 & 8 & 4 \\
3 & $23 / 4$ a 11/05/2018 & 6 & 3 \\
4 & $28 / 05$ a 15/06/2018 & 4 & 3 \\
5 & 9 a 27/07/2018 & 4 & 2 \\
6 & 13 a 31/08/2018 & 3 & 0 \\
\hline Total & & 35 & 18 \\
\hline
\end{tabular}


As sessões com jogos foram aplicadas por profissionais do próprio NIA, com experiência no tratamento de pessoas com dependência em SPAs, dentre os quais a equipe de enfermagem, psicólogos(as) e terapeutas ocupacionais. Esses profissionais foram previamente capacitados pelos pesquisadores, contando com 30 horas de formação inicial (antes da aplicação, sendo 16 horas presenciais e 14 horas a distância) e 10 horas de formação continuada (durante a etapa de aplicação do programa com os adolescentes). Na formação inicial, além do estudo das bases teóricas da metodologia Mind $L a b$, os profissionais vivenciaram o programa que iriam aplicar nos pacientes. A formação continuada foi um espaço de trocas de experiências e de orientações.

O programa com jogos de raciocínio com os adolescentes consistiu em dez sessões de uma hora e meia de duração, cinco vezes por semana (segunda a sextafeira), com duração total de quinze horas. Após o término das dez sessões em cada grupo, a equipe do NIA entrava em contato com os pesquisadores, agendando a aplicação dos testes de avaliação neuropsicológica pós-intervenção em até sete dias. Em função da grade de atividades do NIA e da quantidade de testes e participantes, a coleta era realizada em dois dias consecutivos. Esses dados foram coletados em uma sala dentro do NIA que era disponibilizada pela equipe do local para este propósito. Os testes eram aplicados pelos pesquisadores individualmente e duravam uma média de 90 minutos para a sua aplicação total.

O projeto foi estruturado em quatro eixos de trabalho, a saber:

$1^{\circ}$ eixo: "eu e os problemas" (jogo "Hora do Rush"). O jogo Hora do Rush, é um jogo composto por um tabuleiro que simula um estacionamento, 16 peças que representam miniaturas de carros coloridos e 40 cartas-desafio. Cada carta ilustra uma posição segundo a qual os carros devem ser dispostos no tabuleiro para iniciar o jogo. O objetivo é tirar do "estacionamento" o carro de cor vermelha, sendo que as peças se movimentam apenas para frente ou para trás, na posição em que se encontram (horizontal ou vertical). Trata-se de um jogo de desafio que pode ser jogado individualmente, em duplas ou em grupos pequenos, não se tratando de um jogo de oponentes; $2^{\circ}$ eixo: "eu e o outro" (jogo "Jogo dos 4"). O Jogo dos 4 é um jogo de oponentes composto por um tabuleiro com 16 casas, 8 peças escuras e 8 peças claras, com formatos diferentes (e.g. cilindro, quadrado, mais altas ou mais baixas, entre outros). O objetivo é ser o primeiro jogador a fazer uma sequência ao longo de uma linha, coluna ou diagonal de quatro peças que possuam pelo menos uma característica em comum (por exemplo serem todas quadradas). Para ganhar o jogo, o jogador deve dizer "quatro" para indicar que percebeu a formação de uma sequência.
3० eixo: "eu e a sociedade" (jogos "Forças Unidas e Damas Chinesas"). Forças unidas é um jogo de oponentes composto por um tabuleiro hexagonal, 12 esferas escuras e 12 esferas claras. O jogo tem início com todas as peças no tabuleiro e o objetivo é empurrar seis esferas do oponente para fora do tabuleiro (pela borda) seguindo as regras de movimentação das peças. O jogo Damas Chinesas também é um jogo de oponentes composto por um tabuleiro com 64 casas, 9 peças claras e 9 peças escuras. O jogo tem início com todas as peças no tabuleiro e organizadas de maneira equivalente em dois quadrantes opostos. O objetivo do jogo é mover todas as nove peças para o outro lado do tabuleiro, seguindo as regras de movimentação, até colocá-las na posição em que as peças do oponente começaram o jogo.

4० eixo: "Eu comigo mesmo, com o outro e com a sociedade" (todos os jogos, métodos e estratégias estudados ao longo do processo).

Cada jogo foi utilizado em duas sessões, e em todas elas foram promovidas transcendências, ou seja, reflexões sobre como aplicar as aprendizagens construídas por meio da vivência com os jogos na manutenção da saúde e afastamento das SPAs. Foram ainda promovidos pelos profissionais dois encontros para a retomada dos jogos estudados, com fechamento, reflexões e registro das aprendizagens mais significativas construídas por meio das experiências mediadas pelos jogos: um no meio do processo e outro no final.

Para traçar o perfil dos participantes antes do início da aplicação do programa, a equipe de pesquisadores da Mind Lab aplicou aos adolescentes o Alcohol, Smoking and Substance Involvement Screening Test (ASSIST). Este trata-se de um instrumento para rastreio do uso de SPAs desenvolvido por pesquisadores de vários países sob a coordenação da Organização Mundial da Saúde (OMS)(18), e que já teve a sua validação no Brasil(19). O ASSIST consiste em oito questões sobre o uso e os problemas relacionados a diversas substâncias (tabaco, álcool, maconha, cocaína/crack, anfetaminas ou ecstasy, inalantes, hipnóticos/sedativos, alucinógenos e opioides) e o uso de substâncias injetáveis. Solicitou-se aos participantes que respondessem às perguntas com base nos últimos três meses antes da internação. A pontuação de cada participante nesse instrumento é dada por substância e pode variar de 0 a 31 para o tabaco e de 0 a 39 para as demais substâncias. A pontuação dos participantes nesse instrumento foi classificada de acordo com os critérios de risco para problemas relacionados ao uso de SPAs, incluindo o desenvolvimento de transtornos por uso de substâncias e problemas psicossociais, estabelecidos pela OMS. Os pontos de corte adotados foram: risco baixo (0-10 para álcool e 0-3 para as demais SPAs), risco médio (11-26 para álcool 
e 4-26 para as demais SPAs) e risco alto (27 ou mais) ${ }^{(18)}$. Para informações adicionais sobre o perfil de consumo de SPAs, como a principal droga que levou à internação, foram consultados os prontuários dos participantes.

Para avaliar as funções executivas foram aplicados os instrumentos de avaliação neuropsicológica: Teste de Atenção por Cancelamento (TAC; avaliação da atenção), Teste de Trilhas (atenção e flexibilidade cognitiva) e Torre de Londres ( $T L$; planejamento). Esses instrumentos foram aplicados pelos pesquisadores antes e após a aplicação do programa Mind Lab utilizando jogos de raciocínio.

O Teste de Atenção por Cancelamento (TAC) ${ }^{(20)}$ é composto por três partes. Para cada parte uma matriz diferente é impressa com diferentes tipos de estímulos e a tarefa do participante consiste em assinalar todos os estímulos iguais a um estímulo-alvo anteriormente determinado. A primeira parte do teste avalia a atenção seletiva, que corresponde à capacidade de atentar para um estímulo-alvo dentre outros que são apresentados simultaneamente. O tempo máximo de execução da tarefa é de 1 minuto e o escore máximo de acertos que pode ser atingido é de 50 pontos. A segunda parte do TAC também avalia a atenção seletiva, porém com maior grau de dificuldade. O tempo máximo para essa parte também é de um minuto e o escore máximo que pode ser atingido é de sete pontos. A última parte avalia a atenção alternada, que corresponde à capacidade do participante de mudar o foco da atenção. O tempo máximo para realização dessa parte é de um minuto e o escore máximo é de 52 pontos. Para o presente estudo, foi utilizado o critério de correção e pontuação baseado no número total de acertos menos o número de erros ${ }^{(20)}$, de modo que pontuações mais altas demonstram melhor função da atenção. A avaliação desse instrumento se deu por meio da variação da média das pontuações nos testes em T0 e T1.

O Teste de Trilhas é composto por duas partes (A e $B)^{(21)}$. A parte $A$ avalia a atenção sustentada visual, que se refere à capacidade de manter o foco da atenção ao longo do tempo. Essa parte consiste na apresentação aleatória de doze letras ou números, sendo a tarefa do participante ligá-los em ordem numérica ou alfabética. A parte $B$ avalia a flexibilidade cognitiva, que corresponde à habilidade de alternar a atenção para realizar uma tarefa. Nessa parte do teste são apresentadas, aleatoriamente, letras e números e a tarefa do participante é ligá-los de maneira alternada e em ordem numérica e alfabética (e.g. 1-A-2-B-3-C, e assim por diante). Em ambas as partes, o participante é instruído a realizar as tarefas sem tirar o lápis do papel e o avaliador cronometra o tempo de execução de cada parte. Caso o participante cometa um erro, o avaliador pede que volte ao início da tarefa de modo que quanto mais erros são cometidos, maior será o tempo gasto pelo participante. A avaliação desse instrumento foi realizada com base na média padrão(21), que é estabelecida de acordo com o grau de escolaridade dos participantes. O resultado é dado em pontuação padrão, que corresponde à quantidade de desvio padrão acima ou abaixo da média esperada para aquela escolaridade. Assim, a pontuação mais alta nesse instrumento demonstra melhor atenção sustentada (Parte A) e flexibilidade (Parte B).

O teste da Torre de Londres $^{(22)}$ avalia a função executiva de planejamento, que corresponde à habilidade de planejar ações para atingir um objetivo. O teste é composto por três hastes verticais e três esferas coloridas. $O$ participante deve realizar a tarefa de transposição das três esferas a partir de uma posição inicial fixa, a fim de alcançar as disposições finais especificadas pelo avaliador. Os níveis de dificuldade aumentam gradualmente de acordo com o número de passos necessários para se alcançar a posição final (variando de dois a cinco movimentos). A avaliação desse instrumento considera como resposta correta a solução alcançada pelo participante com o número mínimo de ações, sendo que as respostas podem corresponder a 1, 2 ou 3 pontos, conforme são alcançadas na terceira, segunda ou primeira tentativa, respectivamente. 0 escore máximo possível é de 36 pontos, e quanto maior o escore, melhor o desempenho da função planejamento.

Os dados foram analisados pelo programa IBM SPSS versão $25^{(23)}$. Foi adotado o nível de significância de 0,05. Em função do baixo número de participantes e da não aderência à distribuição normal das variáveis, foi utilizado o Teste de Wilcoxon para amostras pareadas, com o objetivo de verificar possíveis diferenças nas médias nos testes TAC, Trilhas e Torre de Londres em TO e T1. Também foi calculado o tamanho do efeito ( $r$ ) das diferenças significativas entre as médias, cuja intepretação foi: efeito pequeno (de 0,10 a 0,29), efeito moderado (de $0,30$ a 0,49 ) e efeito grande (igual ou maior que 0,5$)^{(24)}$.

A aplicação de um teste de análise de amostras pareadas requer o mesmo número de participantes nos testes pré e pós-intervenção, de modo que só foram considerados para a segunda etapa do estudo os adolescentes que participaram de pelo menos $50 \%$ das oficinas com jogos e que realizaram todos os testes pré e pós-intervenção. Como não houve grupo controle, foi considerado 0 (zero) a variação esperada no resultado dos testes.

O projeto foi aprovado pelo Comitê de Ética em Pesquisa da Faculdade de Ciências da Saúde de São Paulo (CAAE 80249417.6.0000.5399), sob o Parecer no 2.400.515, sendo conduzido de acordo com as Resoluções $466 / 2012$ e 510/2016. O sigilo dos participantes foi garantido durante todo o processo de pesquisa, bem como sua segurança. 


\section{Resultados}

A média de idade dos participantes foi de 15,4 anos, com desvio padrão de 1,37. Quanto à escolaridade, a média foi de 7,4 anos ( $D P=2,27)$. Uma parte expressiva dos participantes vivia na Grande São Paulo (48,5\%), enquanto outra parte estava distribuída no interior do estado de São Paulo (28,6\%) ou era proveniente de outros estados do Brasil (22,9\%)

No que se refere ao perfil de uso de SPAs, observou-se que, entre os 35 adolescentes que participaram da primeira etapa da pesquisa, a maconha e a cocaína foram as substâncias com maior consumo, representando um alto risco para o desenvolvimento de transtornos relacionados ao uso de substâncias e problemas psicossociais, conforme demonstrado na Tabela 2. A pontuação média da maconha foi de 29,6 ( $D P=10,3)$ e da cocaína, de 27,9 ( $D P=12,8)$. A cocaína foi a principal droga que levou à internação dos adolescentes participantes da pesquisa de acordo com os prontuários consultados. Apesar de o ASSIST tratar a cocaína e o crack na mesma categoria, a maior parte dos participantes relatou não ter envolvimento com o uso do crack. Os participantes também relataram uso moderado de outras substâncias (anfetaminas, inalantes hipnóticos, sedativos, alucinógenos). Nenhum participante relatou o uso de substâncias injetáveis.

Tabela 2 - Pontuação e risco por tipo de substância psicoativa de 35 adolescentes internados no CAISM avaliados por meio do ASSIST, no período de 2017 a 2018. São Paulo, SP, Brasil, 2018

\begin{tabular}{lcc}
\hline Substância & M $(\mathbf{D P})^{*}(\mathbf{n}=35)$ & Risco \\
\hline Produtos do tabaco & $22,1(7,8)$ & Médio \\
Álcool & $16,4(12,1)$ & Médio \\
Maconha & $29,6(10,3)$ & Alto \\
Cocaína/crack & $27,9(12,8)$ & Alto \\
Anfetaminas & $9,6(12,9)$ & Médio \\
Inalantes & $11,3(13,2)$ & Médio \\
Hipnóticos, sedativos & $3,6(9,6)$ & Médio \\
Alucinógenos & $4,6(9,8)$ & Médio \\
Opiáceos & $1,4(5,4)$ & Baixo \\
Outras & $0,1(0,3)$ & Baixo \\
\hline
\end{tabular}

"M (DP) = Média e Desvio Padrão

Os resultados das avaliações neuropsicológicas em T0 e T1 estão descritos na Tabela 3.

Tabela 3 - Pontuações em T0 e T1 dos testes de avaliação neuropsicológica, no período de 2017 a 2018. São Paulo, SP, Brasil, 2018

\begin{tabular}{|c|c|c|c|c|c|c|c|}
\hline \multirow{2}{*}{ Instrumento } & \multirow{2}{*}{$\mathbf{n}$} & \multicolumn{2}{|c|}{ Avaliação pré (T0) } & \multicolumn{2}{|c|}{ Avaliação pós (T1) } & \multirow{2}{*}{ p-valort } & \multirow{2}{*}{$\begin{array}{c}\text { Tamanho do } \\
\text { efeito }(r)\end{array}$} \\
\hline & & Mediana & $M(D P)^{*}$ & Mediana & $M(D P)^{*}$ & & \\
\hline $\mathrm{TAC}^{\ddagger}$ & 12 & 49 & $41,1(37,7)$ & 75 & $80,0(24,7)$ & 0,008 & 0,77 \\
\hline$T L^{\S}$ & 17 & 27 & $26,9(5,1)$ & 27 & $27,4(3,2)$ & 0,816 & - \\
\hline Trilhas (parte A) & 18 & $-0,10$ & $-0,46(1,83)$ & $-0,10$ & $0,14(1,71)$ & 0,007 & 0,63 \\
\hline Trilhas (parte B) & 13 & 0,60 & $0,74(0,43)$ & 0,60 & $0,80(0,44)$ & 0,461 & - \\
\hline
\end{tabular}

${ }^{*} M(D P)=$ Média e Desvio Padrão; ${ }^{\top}$ Teste de Wilcoxon Pareado; ${ }^{\ddagger}$ TAC Teste de Atenção por Cancelamento; ${ }^{\S} T L=$ Torre de Londres

No que diz respeito ao TAC, evidenciou-se um aumento na pontuação dos participantes entre T0 e T1 ( $p$-valor $=0,008)$, indicando melhora nessa função executiva. Para essa variável houve um grande tamanho de efeito $(r=0,77)$.

Com relação à função planejamento, avaliada por meio do teste $T L$, observou-se um aumento na pontuação média de 0,5 pontos entre T0 e T1, entretanto, essa variação não foi significativa ( $p$-valor=0,816).

No Teste de Trilhas, parte A, evidenciou-se uma variação média de 0,60 pontos entre as pontuações médias nas avaliações em T0 e T1 ( $p$-valor=0,007), indicando uma melhora na atenção sustentada.

Com relação à função flexibilidade cognitiva avaliada por meio da parte B do Teste de Trilhas, não houve evolução na média entre T0 e T1 ( $p$-valor=0,461), demonstrando que não houve variação significativa dessa variável em T1.

\section{Discussão}

No presente estudo, as substâncias de maior consumo na população participante foram a maconha e a cocaína. De acordo com a classificação proposta pela OMS utilizada nesta pesquisa, o perfil de uso dessas substâncias nos adolescentes participantes é considerado de alto risco para o abuso e dependência de SPAs e problemas psicossociais. Por meio da consulta dos prontuários, a cocaína foi identificada como a principal substância que levou à internação os participantes. Esses achados se contrapõem aos de outras pesquisas, que indicam o álcool como a substância mais consumida no mundo e de destaque entre adolescentes ${ }^{(25-26)}$. Até onde nos foi dado a conhecer, não há estudos com populações em contextos semelhantes que corroboram esses achados, chamando a atenção para as características distintas dos adolescentes em situação de vulnerabilidade e que 
desenvolvem quadros de dependência de SPAs e outros adolescentes que não desenvolvem esses quadros.

Diversos aspectos estão envolvidos no uso de SPAs na adolescência, dentre os quais as intensas mudanças biopsicossociais e o processo de constituição da identidade nesse período, curiosidade, falta de informações, modelos parentais e sociais, mitos e expectativas dos efeitos das substâncias, pressão da indústria e da mídia, além de outros contextos e fatores de risco que merecem destaque e podem ocasionar quadros de dependência(25-26). Para uma compreensão adequada do perfil de uso de SPAs na população estudada é importante ressaltar que o fenômeno da dependência em adolescentes tem nuances e peculiaridades a depender de características individuais, socioeconômicas e demográficas, destacando-se ainda, como fator de risco amplamente discutido na literatura, a falta de amparo das estruturas familiar e social(27-29).

Um estudo apontou para a necessidade do desenvolvimento de programas e estratégias de intervenção eficazes junto ao público adolescente dado o aumento na prevalência dos quadros de dependência em SPAs nessa população, destacando-se a importância de metodologias interventivas lúdicas que, levando em consideração a singularidade e subjetividade do adolescente, bem como os contextos nos quais estão inseridos, possam favorecer a tomada de decisões e escolhas futuras ${ }^{(12)}$. O uso de jogos de raciocínio como recurso terapêutico se insere nessa perspectiva, promovendo o desenvolvimento de habilidades cognitivas, sociais e emocionais capazes de auxiliar nos processos de tomada de decisão, reflexão, planejamento e internalização de atitudes e valores direcionados para a saúde principalmente frente a situações de risco.

O presente estudo destacou que houve melhora na atenção dos adolescentes que participaram do programa com jogos utilizando a metodologia Mind Lab. Esse resultado corrobora os achados de outra pesquisa que utilizou a mesma metodologia com pacientes adultos dependentes de SPAs ${ }^{(10)}$.

Conforme apontado na literatura, déficits e problemas de atenção têm sido associados ao uso de SPAs, particularmente ao uso de cocaína, relacionando-se também a uma maior sensibilidade no sistema de recompensa, de tal forma que há uma tendência desses pacientes a tomar decisões buscando recompensas imediatas e isso pode estar diretamente associado ao uso de SPAs ${ }^{(30-31)}$. Melhoras na função da atenção em pacientes jovens podem, portanto, apresentar uma possibilidade efetiva que os auxilie na tomada de decisões e recuperação do sistema de recompensas e de impulsividade.

Quanto às funções planejamento e flexibilidade cognitiva, embora tenham apresentado melhoras entre a testagem pré e pós-intervenção nos adolescentes participantes deste estudo, estas não foram significativas, necessitando de maiores investigações em estudos futuros. Faz-se importante ressaltar que na metodologia Mind Lab proposta, a utilização dos jogos de raciocínio soma-se ao emprego de métodos metacognitivos e ações de mediação intencionais e planejadas de um profissional da saúde, estimulando a transcendência e generalização da aprendizagem do jogo para reflexões e desenvolvimento de estratégias que podem ser aplicadas na vida cotidiana.

Outros estudos servem de suporte para destacar a possibilidade de utilização dessas estratégias para a reabilitação de funções executivas, além de consistirem em um caminho terapêutico promissor, podendo auxiliar com benefícios em longo prazo e ainda ser facilmente utilizadas em clínicas públicas e particulares no tratamento da dependência de SPAs ${ }^{(32)}$.

\section{Conclusão}

Os resultados permitiram considerar a importância da utilização de jogos de raciocínio com a metodologia Mind Lab no tratamento da população estudada. Foram observadas melhoras nas funções executivas investigadas nos adolescentes que participaram do programa com jogos, ainda que as funções de planejamento e flexibilidade não tenham evidenciado melhoras. A metodologia proposta permitiu o fortalecimento do vínculo entre os pacientes internados e a equipe do serviço, constituindo-se como uma atividade de interesse para os adolescentes, e, por conseguinte, resultando indiretamente numa maior adesão ao tratamento.

O diferencial do presente estudo reside na aplicação prática de novas estratégias e recursos no tratamento de adolescentes com quadro de dependência de SPA. Os resultados encontrados dão subsídio à aplicação de metodologias interventivas lúdicas que favoreçam o desenvolvimento de aspectos cognitivos e sociais, bem como a expressão de sentimentos e reflexão de situações da vida cotidiana.

As limitações deste estudo incluem a seleção não aleatória e limitação no número de participantes, que impedem a generalização dos resultados. Recomenda-se que pesquisas futuras contem com uma população maior a fim de testar a efetividade da metodologia Mind Lab no tratamento de adolescentes dependentes de SPAs. Sugere-se, ainda, a realização de estudos longitudinais para verificar se a melhora em funções executivas pode promover a manutenção da saúde após o período de internação. Por fim, destaca-se a importância de desenvolver pesquisas que proponham intervenções e a verificação de sua efetividade, com vistas a desenvolver metodologias e recursos terapêuticos adequados para o tratamento de adolescentes com quadro de dependência de SPAs. 


\section{Agradecimentos}

A Anita Lilian Zuppo Abed, Itamara Caminada Campello e Ricardo Moino Junior pelo suporte operacional na execução da pesquisa. A Sheila Miguel Basso, Ligia Stella Zito e Tatiana D'Angelo Souza e Silva pela colaboração na fase de coleta de dados.

\section{Referências}

1. Schlindwein-Zanini R, Sotili M. Drug use, Neuropsychological effects and interventions. Cad Bras Saúde Mental. [Internet]. 2019;11(28):94-116. [cited Oct 7 2019]. Available from: http://incubadora.periodicos.ufsc. br/index.php/cbsm/article/view/5592/5439

2. Araújo $M R$, Laranjeira R. Evolução do conceito de dependência. In: Gigliotti A, Guimarães A, editors. Dependência, compulsão e impulsividade. Rio de Janeiro: Rubio; 2016. p. 9-18

3. Pechansky F, Szobot CM, Scivoletto S. Alcohol use among adolescents: concepts, epidemiological characteristics and etiopatogenic factors. Rev Bras Psiquiatr. [Internet]. 2004;26(1):14-7. [cited Oct 9 2019]. Available from: http:// www.scielo.br/scielo.php?script=sci_arttext\&pid=S151644462004000500005\&lng=en

4. Aquino JM de, Teixeira KS, Silva DMR da, Xavier RF, Medeiros SEG, Falcão VTFL. Consumption of alcoholic beverages by students of public schools in the city of Recife-PE. SMAD, Rev Eletrônica Saúde Mental Álcool Drog. [Internet]. 2019;15(2):60-8. [cited Oct 10 2019]. Available from: http://www.revistas.usp.br/smad/article/ view/161577

5. Lopes GM, Nobrega BA, Del Prette G, Scivoletto S. Use of psychoactive substances by adolescents: current panorama. Rev Bras Psiquiatr. [Internet]. 2013;35(1):S51S61. [cited Oct 9 2019]. Available from: http://www. scielo.br/scielo.php?script=sci_arttext\&pid=S151644462013000500007\&lng=en

6. Blakemore SJ. Imaging brain development: the adolescent brain. Neuroimage. 2012 Jun;61(2):397-406. doi: http://doi.org/10.1016/j.neuroimage.2011.11.080 7. Squeglia LM, Jacobus J, Tapert SF. The influence of substance use on adolescent brain development. Clin EEG Neurosci. 2009 Jan;40(1):31-8. doi: http://doi. org/10.1177/155005940904000110

8. Clark DB, Chung T, Thatcher DL, Pajtek S, Long EC. Psychological dysregulation, white matter disorganization and substance use disorders in adolescence. Addiction. 2012 July;107(1):206-14. doi: http://doi. org/10.1111/j.1360-0443.2011.03566.x

9. Clark DB, Chung T, Martin CS, Hasler BP, Fitzgerald DH, Luna $B$, et al. Adolescent Executive Dysfunction in Daily Life: Relationships to Risks, Brain Structure and Substance Use. Front Behav Neurosci. 2017 Nov;11:223. doi: http:// doi.org/10.3389/fnbeh.2017.00223
10. Abed ALZ, Garcia SRR, Duarte CE, Amaral SS, Oda $A L$, Pantano T. The impact of the use of thinking games, meta-cognitive methods and planned interventions in the executive functions of chemically dependent people. Rev Bras Neurol Psiquiatr. [Internet]. 2018;22(3):22643. [cited Oct 9 2019]. Available from: https://www. revneuropsiq.com.br/rbnp/article/view/236/164

11. Takesian AE, Hensch TK. Balancing plasticity/stability across brain development. Prog Brain Res. 2013;207:3-34. doi: http://doi.org/10.1016/B978-0-444-63327-9.00001-1 12. Silva ML da, Rosa SS. Games and music: Therapeutic occupational resources in the treatment of teenagers users of psychoactive substances. Adolesc Saúde. [Internet]. 2017;14(4):58-65. [cited Out 6 2019]. Available from: http://hdl.handle.net/11449/175688

13. Campbell DT, Stanley JC. Experimental and quasiexperimental designs for research. In: Gage NL, editor. Handbook of research on teaching. Chicago: Rand McNally; 1963. p. 1-76

14. Oliveira MS, Szupszynski K, DiClemente C. Study of motivation for treatment in teenagers users of illicit substances. Psico. [Internet]. 2010;41(1):40-6. [cited Oct 7 2019]. Available from: http://revistaseletronicas. pucrs.br/revistapsico/ojs/index.php/revistapsico/article/ view/7207/5215

15. Scaduto AA, Barbieri V. The discourse about adherence of chemically dependent adolescents to treatment in a public health institution. Ciênc Saúde Coletiva. 2009 Apr;14(2):605-14. doi: http://doi.org/10.1590/ S1413-81232009000200029

16. Andretta I, Limberger J, Oliveira MS. Treatment's dropout of substance abuse adolescents who committed an infraction. Aletheia. [Internet]. 2014;43(44):11628. [cited Oct 9 2019]. Available from: http://pepsic. bvsalud.org/scielo.php?script=sci_arttext\&pid=S141303942014000100009\&Ing=pt

17. Gonçalves JRL, Canassa LW, Cruz LC, Pereira AR, Santos DM, Gonçalves AR. Adherence to treatment: perception of adolescents in chemical dependency. SMAD, Rev Eletrôn Saúde Mental Álcool Drog. [Internet]. 2019;15(1):57-83. [cited Oct 10 2019]. Available from: https://www.redalyc. org/articulo. oa?id=80358712008

18. World Health Organization. The Alcohol, Smoking and Substance Involvement Screening Test (ASSIST): manual for use in primary care [Internet]. Switzerland: WHO Library Cataloguing-in-Publication Data; 2010. [cited Mar 17 2019]. Available from: https://www.who. int/substance_abuse/publications/assist/en/

19. Henrique IFS, De Micheli D, Lacerda RB, Lacerda LA, Formigoni MLOS. Validation of the Brazilian version of Alcohol, Smoking and Substance Involvement Screening Test (ASSIST). Rev Assoc Med Bras. [Internet]. 2004;50(2):199-206. [cited Oct 9 2019]. Available 
from: http://www.scielo.br/scielo.php?script=sci_ arttext\&pid=S0104-42302004000200039\&lng=en

20. Montiel J, Capovilla A. Avaliação da atenção: Teste de Atenção por Cancelamento. In: Capovilla A, Capovilla FC. Teoria e pesquisa em avaliação neuropsicológica. São Paulo (SP): Memmon; 2007. p. 119-24

21. Montiel J, Capovilla A. Teste de trilhas. In: Capovilla A, Capovilla FC. Teoria e pesquisa em avaliação neuropsicológica. São Paulo (SP): Memmon; 2007. p. 94-5 22. Menezes A, Dias NM, Godoy S, Seabra AG. Teste da Torre de Londres e avaliação do planejamento executivo em adolescentes brasileiros. Psicologia Educação e Cultura. [Internet]. 2011;15(2):367-37. [cited Aug 16 2020]. Avaliable from: http://pec.ispgaya.pt/index.php/ publicacoes/40-volume-xv-n-2/669-teste-da-torre-deIondres-e-avaliacao-do-planejamento-executivo-emadolescentes-brasileiros

23. IBM Corp. IBM SPSS Statistics for Windows, version 25.0. NewYork: IBM Corp; 2017

24. Howell DC. Statistical methods for psychology. $7^{\text {th }}$ ed. Belmont (CA): Wadsworth, Cengage Learning; 2010. $770 \mathrm{p}$ 25. Filho EAF. Profile of alcohol and drug use in adolescent pupils in a Brazilian state capital. SMAD, Rev Eletrôn Saúde Mental Álcool Drog. [Internet]. 2014;10(2):78-4. [cited Oct 14 2019]. Available from: http://www.revistas.usp. br/smad/article/view/98722

26. Tavares MLO, Reinaldo AMS, Villa EA, Pereira MO, Monteiro MAM. Information, beliefs and attitudes of schoolchildren about the use of alcohol and other drugs. SMAD, Rev Eletrônica Saúde Mental Álcool Drog. [Internet]. 2019;15(2):45-51. [cited Oct 14 2019]. Available from: https://www.revistas.usp.br/smad/article/view/161572

27. Pechanscky F, Von Diemen L, De Micheli D, Amaral MB do. Fatores de risco e proteção em diferentes grupos de usuários: adolescentes, idosos, mulheres e indígenas. In: Duarte PCAV, Formigoni MLOS, organizadores. O uso de substâncias psicoativas no Brasil: módulo 1. [Internet]. Brasília: Secretaria Nacional de Políticas sobre Drogas; 2017. p. 71-92. [Acesso 16 out 2019]. Available from: http://hdl.handle.net/10183/198390

28. Castro LM. Adolescent's expressions about illicit drug use. Revista Ciencias Sociales (Cr) [Internet]. 2009; III(123-124):119-37. [cited Oct 2 2019]. Available from: https://www.redalyc.org/articulo.oa?id=15313756008

29. Schenker M, Minayo MCS. Risk and protective factors and drug use among adolescence. Ciênc Saúde Colet. [Internet]. 2005;10(3):707-17. [cited Sep 26 2019]. Available from: http://www.
scielo.br/scielo.php?script=sci_arttext\&pid=S141381232005000300027\&lng=en

30. Cancian A, Germano L, Cerutti F, Oliveira M. Attention deficit hyperactivity disorder and crack cocaine. SMAD, Rev Eletrônica Saúde Mental Álcool Drog. [Internet]. 2018;13(2):78-85. [cited Oct 16 2019]. Available from: http://www.revistas.usp.br/smad/article/view/149361

31. Balconi M, Finocchiaro R, Campanella S. Reward sensitivity, decisional bias, and metacognitive deficits in cocaine drug addiction. J Addict Med. [Internet]. 2014;8(6):399-406. [cited Oct 2019]. Available from: https://www.ncbi.nlm.nih.gov/pubmed/25303980

32. Unterrainer JM, Kaller CP, Halsband $U$, Rahm B. Planning abilities and chess: a comparison of chess and non-chess players on the Tower of London task. Br J Psychol. 2006 Aug;97(3):299-311. doi: http://doi. org/10.1348/000712605X71407

\section{Contribuição dos autores}

Concepção e desenho da pesquisa: Cláudio Elias Duarte, Sandra Regina Rezende Garcia, Sarah Virginia dos Santos Braga, Felipe Salles Neves Machado, Jefferson Luiz Pereira, Sofia Marques Viana Ulisses. Obtenção de dados: Cláudio Elias Duarte, Sandra Regina Rezende Garcia, Sarah Virginia dos Santos Braga, Felipe Salles Neves Machado, Sofia Marques Viana Ulisses. Análise e interpretação dos dados: Cláudio Elias Duarte, Sandra Regina Rezende Garcia, Sarah Virginia dos Santos Braga, Felipe Salles Neves Machado, Jefferson Luiz Pereira, Sofia Marques Viana Ulisses. Análise estatística: Jefferson Luiz Pereira. Obtenção de financiamento: Cláudio Elias Duarte, Sandra Regina Rezende Garcia. Redação do manuscrito: Sofia Marques Viana Ulisses, Jefferson Luiz Pereira. Revisão crítica do manuscrito quanto ao conteúdo intelectual importante: Cláudio Elias Duarte, Sandra Regina Rezende Garcia, Sarah Virginia dos Santos Braga, Felipe Salles Neves Machado.

Todos os autores aprovaram a versão final do texto.

Conflito de interesse: os autores declararam que não há conflito de interesse. 